\title{
RF Impedance Probe Measurements of Ionospheric Electron Densities
}

\author{
J. A. Kane, J. E. Jackson, and H. A. Whale \\ Contribution From Goddard Space Flight Center, Greenbelt, Md.
}

(Received March 29, 1962; revised May 31, 1962)

\begin{abstract}
The Aerobee-Hi rocket, NASA 4.07, obtained vertical electron density profiles in the ionosphere simultaneously by the Seddon $\mathrm{CW}$ propagation technique and by an $\mathrm{RF}$ impedance probe technique. The experimental goal was to assess the performance of the $\mathrm{RF}$ probe against the accurate values from the $\mathrm{CW}$ method. In the RF probe method, the electron density $N$ was derived from the dielectric constant $K$ of the medium at a frequency $f=7.75 \mathrm{Mc} / \mathrm{s}$. If the earth's magnetic field is neglected, the relation between these quantities may be given as $K=1-\left(81 N / f^{2}\right)$. An expression for the dielectric constant including the effects of the earth's magnetic field is derived. The fractional error introduced by neglecting the earth's field is of the order of $\left(f_{E} / f\right)^{2}$ where $f_{H}$ is the electron gyrofrequency.

It was concluded that, with allowance for the positive ion sheath around the rocket, the $\mathrm{RF}$ impedance probe can yield reliable values of electron density. These values were obtained from changes in the capacitive part of the probe's impedance. Small, apparently anomalous changes in the resistive part were also observed.
\end{abstract}

\section{Introduction}

The most accurate electron density profiles of the ionosphere have been obtained by Seddon's CW propagation method [Seddon, 1953], which yields excellent results for rocket soundings in a fairly quiet, horizontally uniform ionosphere, particularly if corrections are made for obliquity effects [Jackson, 1957]. However, in order that synoptic measurements may be made under all ionospheric conditions, other techniques are required. The RF probe technique discussed in the present paper was developed to provide such a method. It is based on the results of several previous rocket flights [Jackson and Pickar, 1957] in which the ionosphere was observed to have an effect on the impedance of rocket-borne antennas. The antenna impedance was determined during these earlier rocket flights by monitoring the voltages on both sides of the matching network between the antenna and the CW transmitter. With the aid of pre-flight calibrations, these two sets of observations were sufficent to determine both the reactive and the resistive components of the antenna impedance, but it was not possible to relate the observed impedance variations to ambient ionospheric parameters until the proper precautions were taken to minimize contamination of the rocket environment. When this was effected (mainly by sealing the rocket fuel tanks after the completion of propulsion), it was found that electron densities derived from the impedance observations showed very good correlation with the $\mathrm{CW}$ propagation results [Jackson and Kane, 1959].

Since the antennas used for the RF impedance probe measurenents were electrically short, their impedances were primarily capacitive. This capacitance changed by an amount proportional to the ambient electron density. In these experiments electron densities were obtained by attributing the capacitance changes to changes in the dielectric constant $K$ of the medium and then calculating the electron density from the relation:

$$
K=1-\frac{81 N}{f^{2}}
$$

where $N$ is the density in electrons $/ \mathrm{cm}^{3}$ and $f$ is the operating frequency in $\mathrm{kc} / \mathrm{s}$.

In past experiments the electron density values derived from eq (1) had been too small by a factor of about 3. For some applications this could be considered fortunate, since it indicated that the impedance of a transmitting antenna was affected less by the ionosphere than might have been expected from the ambient ionization. It was tentatively concluded from these early results that the ionospheric effects were reduced, primarily by distortion of the antenna environment due to the large RF amplitude (in the order of $200 \mathrm{v}$ ) required for the $\mathrm{CW}$ propagation experiment. The rocket experiment described in this paper was performed to see if the accuracy of the $\mathrm{RF}$ probe would be substantially improved by making the measurements at low RF voltages. A preliminary account of the results of this experiment has been given by Jackson and Kane [1960].

In order to compare the electron density values obtained from the RF probe with the actual densities, a CW propagation experiment was performed during this rocket flight at 7.75 and $46.5 \mathrm{Mc} / \mathrm{s}$ on a timesharing basis. To fulfill the requirements of both 
experiments, the amplitude of the fixed-frequency $7.75-\mathrm{Mc} / \mathrm{s}$ signal applied to the antenna was cycled periodically through the following levels: $0.2,2,2$, and $200 \mathrm{v}$, each with a duration of 1 sec. When the nominal free-space RF amplitude of $200 \mathrm{v}$ was on the antenna, the CW propagation experiment was performed to establish the reference electron density profile; the $0.2-\mathrm{v}$ and $2-\mathrm{v}$ levels were used for the RF probe experiments. Thus, the CW propagation experiment was performed during 1 sec out of every four, giving approximately one value of electron density every $4 \mathrm{~km}$ in the region of interest. The CW propagation experiment was thus slightly handicapped, since there were not quite enough data points to plot the curvature of the resulting profile precisely. This caused the profile to appear somewhat jagged; nevertheless, it was accurate enough to assess the quality of the RF probe data.

The vertical-incidence ionospheric sounding station located near the launching site provided additional support for the RF probe experiment. A quiet ionosphere was desired in order to optimize the measurements made by the $\mathrm{CW}$ propagation experiment; therefore, continuous monitoring of the ionosphere was conducted prior to the firing to insure that this requirement would be met. Also, with the experiment performed under quiet conditions, the $\mathrm{P}^{\prime}-f$ records obtained could readily be analyzed and a third independent determination of the electron density profile thereby obtained.

Finally, comparison experiments such as Langmuir probe, ion trap, and electric field meter measurements were included in the rocket, making it possible to acquire further data for correlation purposes.

\section{Electron Densities From the CW Propagation Experiment}

In the CW propagation experiment two phasecoherent signals, at $7.75 \mathrm{Mc} / \mathrm{s}$ and $46.5 \mathrm{Mc} / \mathrm{s}$, were transmitted from the rocket and received on the ground with circularly polarized antennas. The instrumentation at the receiving station [Jackson and Spaid, 1959] provided data for both the ordinary and the extraordinary components of the $7.75 \mathrm{Mc} / \mathrm{s}$ signals. Since the electron density could be derived from either the ordinary or the extraordinary ray data, this experiment provided two independent measurements of the electron density profile. The two curves are shown in figure 1, where electron density is plotted against the time after launching. (The peak of the flight occurred about 250 sec after launch time.) Results obtained from this type of measurement are most accurate when the radial velocity of the rocket from the receiving site is large and the transverse velocity small. Near the peak the radial velocity is small, and any changes in the ionization between the rocket and the receiving station may appear as large apparent changes in the electron density at the rocket. This effect can be observed in the large fluctuations in the results between 200 and 300 sec (fig. 1).

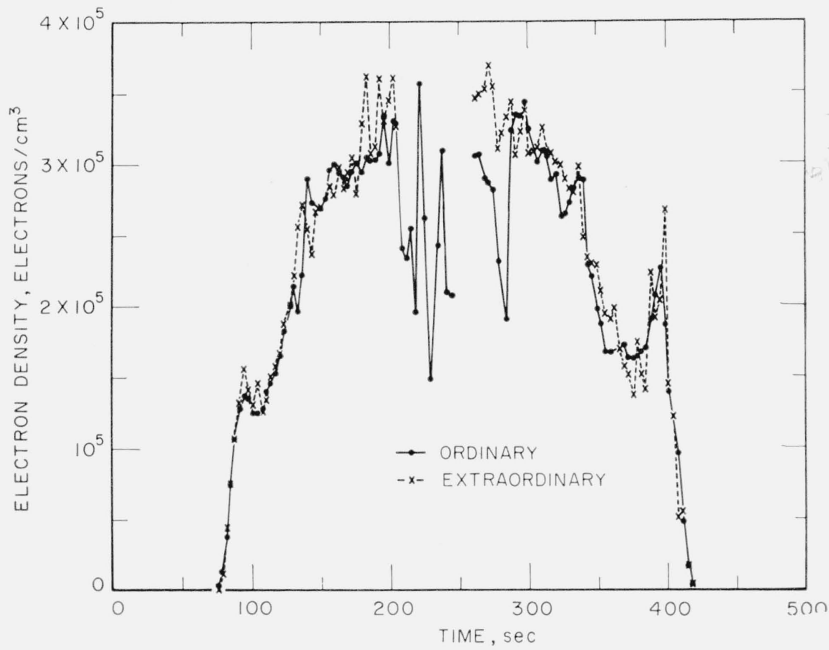

Figure 1. Electron density versus flight time derived from propagation data on ordinary and extraordinary polarization modes.

1128 CST, Sept. 14, 1959, Ft. Churchill, Manitoba, Canada.



Figure 2. Altitude dependence of the mean electron density derived from the propagation experiment during rocket ascent and descent.

1128 CST, Sept. 14, 1959, Ft. Churchill, Manitoba, Canada.

If the data for the 200-300 sec period are disregarded, there is good agreement between the electron densities obtained from the two polarization components. The slight differences that are noticeable are due in part to the fact that the ordinary and the extraordinary rays followed slightly different paths in the ionosphere and were affected differently by irregularities and changes in the region between the rocket and the receiver. Thus a mean value of the two curves should be a slightly better measure 
of the electron density at the rocket than that obtained from either ray by itself. Such a mean curve is plotted in figure 2 , where the electron density $\mathrm{s}$ shown as a function of altitude for the ascending ( oolid line) and descending (dashed line) parts of the rocket trajectory. From this comparison it can be seen that the measured electron densities differed by an appreciable factor for the ascending and the descending parts of the flight. The difference is most noticeable in the vicinity of $120 \mathrm{~km}$ where descent values were about 50 percent greater than the ascent values. This indicates the presence of horizontal gradients, whose presence reduces the accuracy of the obliquity correction required to derive the descent values.

\section{The RF Probe}

As was indicated earlier, the sensor used in the $\mathrm{RF}$ probe experiment is a capacitor whose dielectric constant is that of the ambient medium. Configurations which could be used for this capacitor include parallel plates, collinear cylindrical whips, and insulated sections of a rocket surface. In the experiment under discussion the sensor was a rocketborne $7.75 \mathrm{Mc} / \mathrm{s}$ antenna consisting of two $3-\mathrm{m}$ collinear whips. Because of its short electrical length, the impedance of this antenna at $7.75 \mathrm{Mc} / \mathrm{s}$ was primarily capacitive.

Since the method formerly used for impedance measurements (monitoring the input and output of the matching network) was found to have poor resolution and to suffer from drifts in the calibration curve, an improved method was developed for the NASA 4.07. This method can be understood from figure 3, which shows the circuit and responses of the RF probe. The circuit consisted of a lowimpedance $7.75 \mathrm{Mc} / \mathrm{s}$ signal source and a matching network connecting this source to the probe. The impedance probe, together with its associated network, exhibited a parallel resonance for the proper value of the variable capacitor $C-C$. The circuit was designed so that resonance would occur near the low capacitance end of the capacitor when the probe was in a nonionized madium. In the ionosphere, the capacitance of the probe decreased because of the presence of free electrons, and additional capacitance had to be provided by the capacitor $C-C$ to achieve parallel resonance.

The amplitude of the RF signal appearing at the probe was detected at the point $A$ (fig. 3) and telemetered to the ground. In operation, the capacitor C-C was rotated continuously, causing the $\mathrm{RF}$ response of the probe circuit to vary in the manner indicated by the responses shown in the figure. The probe capacitance determined the position of the response peaks whose amplitude indicated the resistive loading. The dielectric constant of the medium was calculated from this capacitance and then used to calculate electron densities with the aid of eq (1). The error inherent in eq (1), as a result of neglecting the effect of the earth's magnetic field, was at most 4 percent (see appendix).
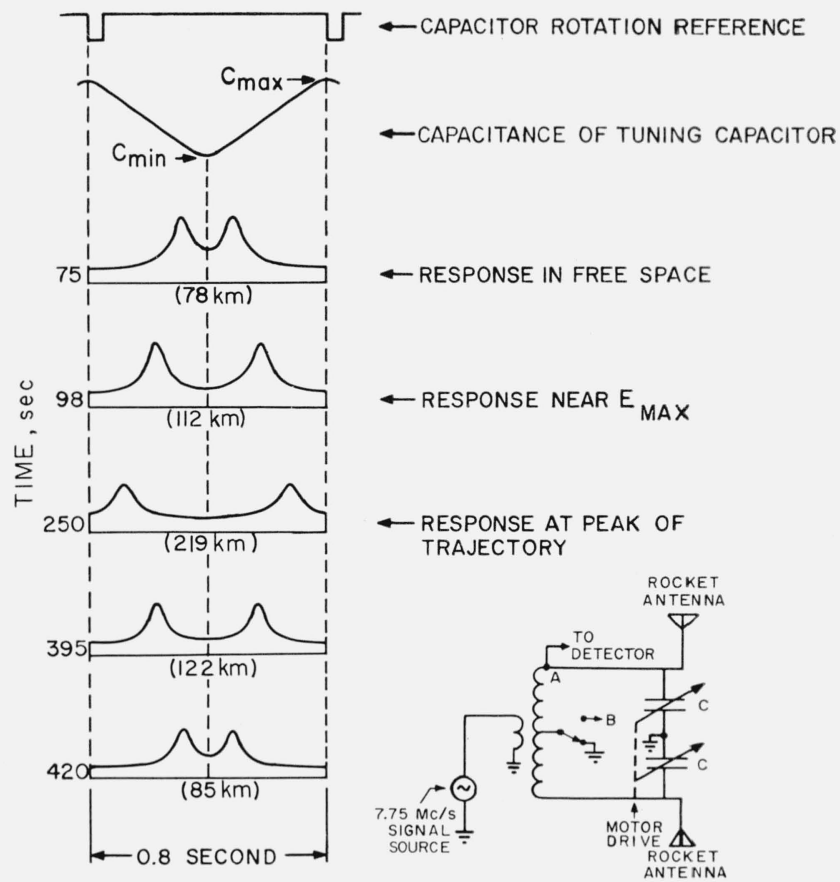

$\leftarrow$ RESPONSE IN FREE SPACE

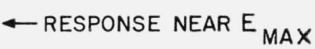

Figure 3. Response curves of the RF probe circuit.

1128 CST, Sept. 14, 1959, Ft. Churchill, Manitoba, Canada.

As mentioned earlier, a four-step time-sharing sequence was used. The antenna probe was connected to the variable condenser $C-C$ during the first two steps, when the nominal RF voltage levels were respectively 0.2 and $2 \mathrm{v}$. For the third and fourth steps, the variable condenser was disconnected from the antenna probe and replaced by a fixed condenser selected to give resonance under freespace conditions. During the third step a nominal voltage of $2 \mathrm{v}$ was used and the amplitude was again monitored at point $A$. This provided a means for detecting small irregularities which could have occurred during the 1-sec periods allotted for each step of the sequence. During steps 1, 2, and 3, the center tap of the matching transformer (fig. 3) was connected to ground. During the fourth step a nominal voltage of $200 \mathrm{v}$ was applied to the probe, which was then used as the low-frequency transmitting antenna for the CW propagation experiment. In this case the fixed capacitor was used instead of the variable capacitor $C-C$ in order to prevent large variations in amplitude and phase at the lowfrequency antenna. Either of these variations would have introduced severe complications into the CW propagation experiment. To permit detection of a possible collection of electrons by the transmitting antenna, the center tap of the matching network was disconnected from the ground during the fourth step of the sequence and connected to a current-measuring circuit. The antenna voltage was also monitored during this fourth step to indicate effects of the ionosphere upon the antenna impedance while the $\mathrm{CW}$ propagation experiment was in progress. 


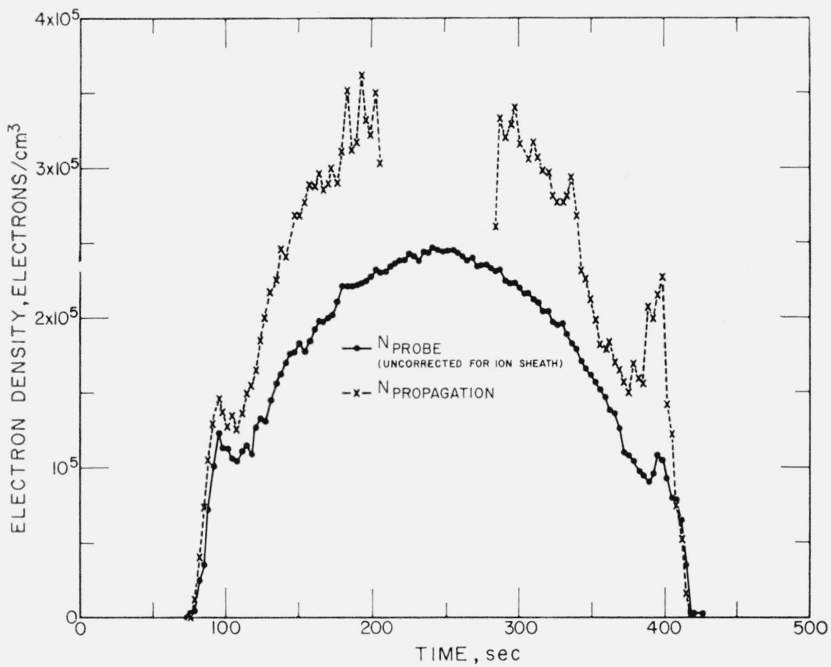

FIgure 4. Comparison of electron density versus flight time for both the $R F$ probe and $C W$ propagation methods.

1128 CST, Sept. 14, 1959, Ft. Churchill, Manitoba, Canada.

Briefly, the experimental results of the RF probe observations were as follows:

(1) During the propagation experiment (with 200 $v$ on the antenna) the effect of the ionosphere upon the antenna capacitance was only 30 percent of that calculated on the basis of ambient electron density. This result is in agreement with the previous observations made under similar conditions. During this last sequence a large electron current was collected by the antenna in the altitude range from 60 to $110 \mathrm{~km}$, which is the region where electrical breakdown occurs [Jackson and Kane, 1959]. However, above the breakdown region the current collected by the antenna was below the $10^{-8}$ amp detection sensitivity of the current-monitoring circuit.

(2) No irregularities were indicated by the observations made during the third step of the programing sequence.

(3) The electron densities calculated from the response curves obtained during the first and second steps lie in the range 70 to 80 percent of the actual values. Therefore it is apparent that a substantial improvement in the accuracy of the RF probe was achieved by performing the measurements at low $\mathrm{RF}$ levels. The remaining difference between the $\mathrm{RF}$ probe and $\mathrm{CW}$ propagation techniques will be discussed in the section on the ion sheath. The electron densities derived from the responses obtained at the $0.2-\mathrm{v}$ level are plotted against flight time as the solid curve in figure 4. Impedance measurements at $2.0 \mathrm{v}$ yielded a profile only a few percent lower than the $0.2-\mathrm{v}$ profile. The values obtained from the propagation method (mean of the ordinary and extraordinary ray results) are also plotted in figure 4. It should be noted that, in contrast to the $\mathrm{CW}$ propagation data, the $\mathrm{RF}$ probe data experienced no degradation during the time interval from 200 to 300 sec. The electron density derived from the RF probe measurements is plotted as a function of altitude in figure 5. It is of interest

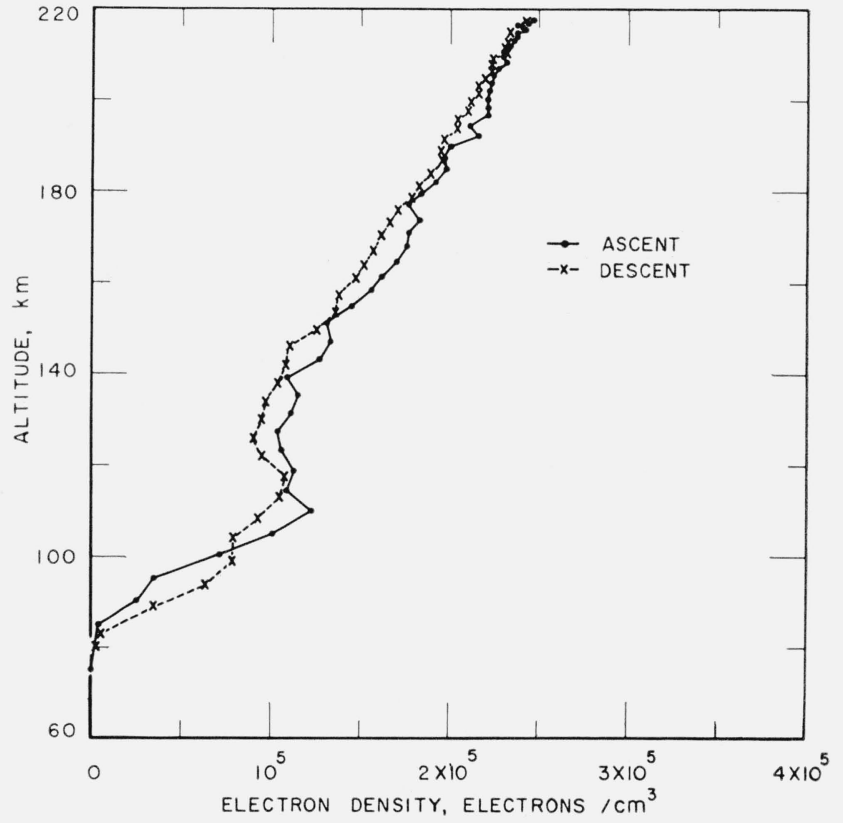

Figure 5. Comparison of electron density obtained by $R F$ probe during rocket ascent and descent.

1128 CST, Sept. 14, 1959, Ft. Churchill, Manitoba, Canada.

to note that the absence of a deep "valley" in the daytime above the maximum of the $E$ region was, in this instance, verified by a direct measurement.

(4) A comparison between the profiles derived from the $\mathrm{RF}$ probe, the $\mathrm{CW}$ propagation method experiment, and the $P^{\prime}-f$ record is given in figure 6 . The $P^{\prime}-f$ record obtained during the flight is shown in figure 7. This ionogram was analyzed by means of a five-point reduction process based upon coefficients obtained by extrapolating Schmerling's [1958] values to those appropriate in the latitude of Fort Churchill. As had been expected, the electron density values calculated from the $P^{\prime}-f$ record were in good agreement with the values obtained by the $\mathrm{CW}$ propagation method during the upward part of the flight.

\section{Ion Sheath}

The difference between the two methods on the question of the absolute values of the electron density will now be considered (fig. 4). This difference is to be expected since, in the ionosphere, the rocket body and antennas will normally be enclosed in an ion sheath. If the photoelectric effect can be ignored, the rocket body and antennas will acquire a net negative charge due to the difference between the electron and positive ion velocities. The effect of this surface charge is to create in the adjacent medium an electric field that depletes the electron content over a distance which can be represented as an equivalent sheath thickness. The rocket potential $\Phi$ is determined by the condition that the positive ion flux to the rocket surface is equal to the flux of electrons with energies greater than $e \oint$ (where $e$ is the charge on an electron). This process has been discussed by Jastrow and Pearse [1957]. 
disagreement. This discrepancy is not unreasonable in view of the nature of the approximations used to compute the probe correction factor. In particular, complicated geometry near the antenna base allows a small amount of stray capacitance to be entirely within the sheath. The effect of this parallel stray capacitance is essentially to magnify the probe correction factor by $1+\left(C_{B} / C_{0}\right)$, where $C_{B} / C_{0}$ is the ratio of this stray capacitance to the total free-space antenna capacitance. Although the precise value of $C_{B} / C_{\mathrm{E}}$ is unknown, it is probably less than 0.2 ; and, since it should be proportional to the sheath dimension, it should decrease with increasing altitude. It will be noted, however, that the difference between the propagation results and the corrected probe results (table 1) shows a somewhat different altitude variance than does the sheath dimension. This could be due to electron depletion by attachment to molecules coming from a small leak in some pressurized compartment of the rocket (most likely a small leak in the valve used to seal the rocket fuel tanks). The supposed leakage proceeds by effusion above 100 $\mathrm{km}$ and would therefore be essentially constant over the short time interval of the rocket flight. Such a mechanism could also affect the Langmuir probe results.

It is hoped that some of these questions will be resolved in the next rocket flight in which it is planned to repeat this experiment with the following refinements: (1) a guard ring arrangement will be provided to eliminate the uncertainties associated with the antenna base geometry; (2) the Langmuir probe measurements will be performed with higher resolution; (3) a d-c voltage will be programed on the antenna to vary the sheath dimension; (4) the RF impedance measurement will be performed at two exploring frequencies to determine the effective sheath capacitance; and (5) the RF probe sampling rate will be increased by a factor of at least 10 .

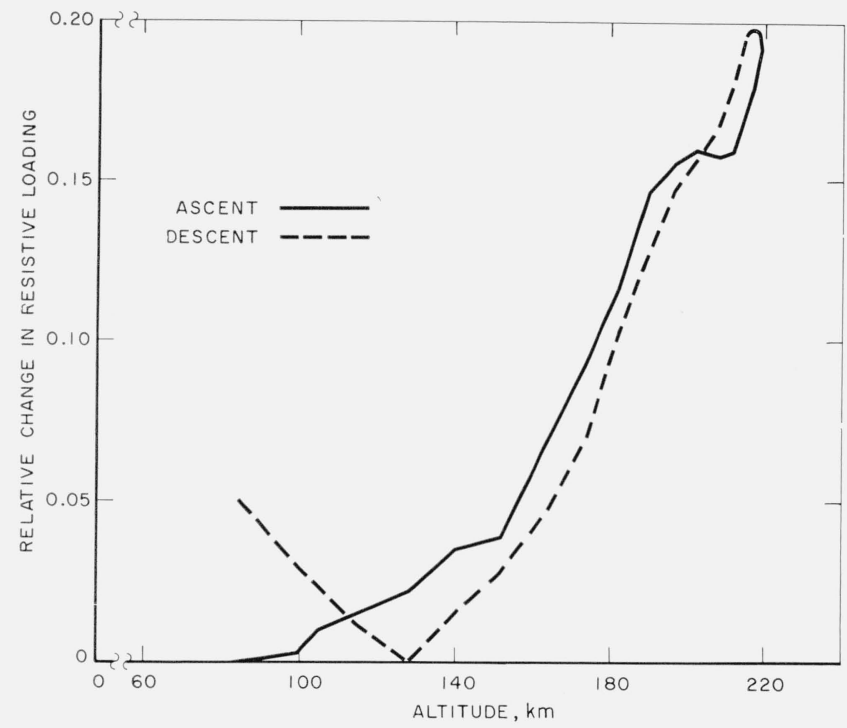

Figure 8. Relative change in resistive loading of $R F$ probe versus altitude.

1128 CST, Sept. 14, 1959, Ft. Churchill, Manitoba, Canada.

\section{Resistive Loading of the Probe}

In addition to measuring the reactive component, the method described above also gives the resistive component of the antenna impedance: The latter can be determined from the amplitude of the response curve. During the flight considered here the variation of the amplitude was small. There was also a small, slow drift, probably arising from the slow decay of the battery voltage during the flight. A linear correction has been applied to the measured amplitudes to compensate for this steady drift. The results are shown in figure 8 , where the relative change in the resistive loading is plotted as a function of altitude. The following mechanisms, which refer to the case where the magnitude of the RF field is at most comparable to the d-c rocket potential, have been examined in an attempt to explain this resistive loading.

\subsection{Radiation Resistance}

The radiation resistance of a short linear antenna in free space is given by the rather well known expression

$$
R=20 \pi^{2}\left(\frac{l}{\lambda_{0}}\right)^{2} \text { ohms }
$$

where $\left(l / \lambda_{0}\right)$ is the ratio of antenna length to free-space wavelength. It is a straightforward matter to show that in the ionosphere the radiation resistance becomes

$$
R=2 C \pi^{2}\left(\frac{l}{\lambda_{0}}\right)^{2} \sqrt{K} \text { ohms. }
$$

This mechanism by itself gives a resistive loading proportional to $\sqrt{K}$ and thus predicts, for the amplitude of the response curve, an altitude variation which is opposite to that observed.

\subsection{Collision Frequency Damping}

The electron collision frequency normally is a decreasing function of altitude. This would not necessarily be true if the electron could gain energy from the antenna field as the electron mean free path increased with altitude. However, above $100 \mathrm{~km}$ this process is unlikely since the electron mean free path is greater than $10 \mathrm{~cm}$, and, under the conditions of this experiment, the maximum oscillation amplitude of an electron under the influence of the antenna field is $0.5 \mathrm{c.m}$.

\subsection{Inelastic Electron Collisions With the Antenna Surface}

As has been pointed out, the vehicle potential is maintained by the flux of electrons with energies greater than $e \oint$, where $\oint$ is the rocket potential. This electron flux has also an additional energy component derived from the RF field of the antenna, and the dissipation of this component by the electrons captured on the antenna surface represents a resistive loading on the antenna. A calculation of the effect shows that this mechanism produces a resis- 
tive loading of the proper altitude dependence but approximately one order of magnitude smaller than that observed.

\subsection{Excitation of Electro-Acoustical Waves}

Another resistive loading factor might arise from the production of an electron pressure wave due to interaction of the $\mathrm{RF}$ field and the antenna ion sheath. Such a wave might absorb energy and thus contribute to the resistive loading. This mechanism is considered more fully in a separate paper [Whale, $1962]$.

\section{Effect of Irregularities in the Ionosphere}

In the case of an oblique rocket trajectory, the accuracy of electron density profiles obtained with the propagation experiment is limited by the assumption that the ionosphere is horizontally uniform.

The effects of some nonuniformity can be seen very easily when the apparent electron density obtained from the propagation experiment is plotted as a function of the apparent electron density obtained from the RF probe (fig. 9). These RF probe results are not corrected for the presence of the ion sheath, since this correction would introduce only a relatively constant multiplying factor to the results. For the ascent of the rocket, the points

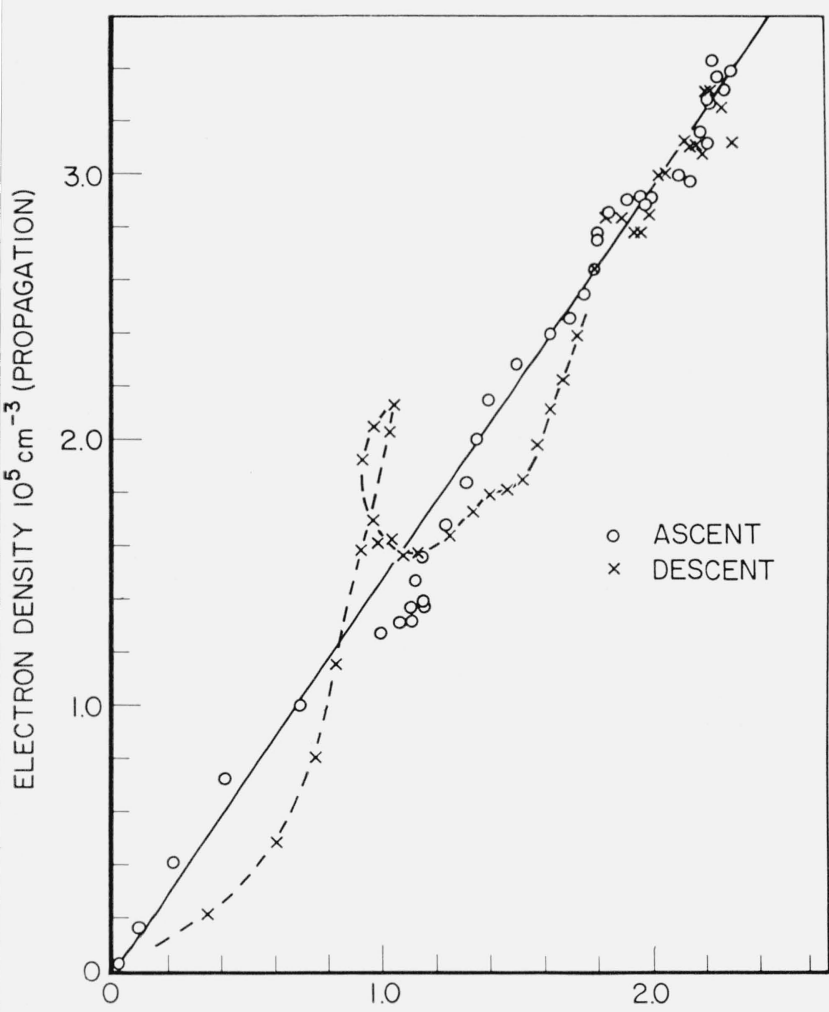

APPARENT ELECTRON DENSITY $10^{5} \mathrm{~cm}^{-3}$ (PROBE)

Figure 9. Propagation results versus $R F$ probe results illustrating the effect of an ionospheric irregularity.

1128 CST, Sept. 14, 1959, Ft. Churchill, Manitoba, Canada. fall on a fairly smooth curve which can be represented reasonably well by the straight line shown. However, the descent curve shows a loop which indicates that some irregularity was encountered on this part of the trajectory.

\section{Conclusions}

The development of a reliable RF impedance probe has been considerably advanced by the NASA 4.07 rocket flight. The probe, in its present form, together with a separate measurement of the vehicle potential, is sufficient to determine electron densities to an accuracy of the order of 25 percent. Further improvements in the technique are under development.

\section{Appendix. The Dielectric Constant of an Ionized Medium}

For an ionized gas under the influence of a steady magnetic field, the dielectric constant becomes a tensor [Allis, 1956]. If a rectangular coordinate system is chosen with the $Z$-axis in the direction of the magnetic field this tensor has the form

$$
K=\left|\begin{array}{ccc}
K_{T} & j K_{H} & 0 \\
-j K_{H} & K_{T} & 0 \\
0 & 0 & K_{p}
\end{array}\right|
$$

where

$$
\begin{aligned}
K_{p} & =1-X \frac{1}{1-j Z}, \\
K_{T} & =1-X \frac{1+j Z}{(1+j Z)^{2}-Y^{2}}, \\
K_{H} & =X \frac{Y}{(1+j Z)^{2}-Y^{2}}, \\
X & =\left(\frac{f_{p}}{f}\right)^{2}, \\
f_{p} & =\text { plasma frequency, } \\
f & =\text { exploring frequency, } \\
Y & =\frac{f_{H}}{f}, \\
f_{H} & =\text { gyrofrequency of the electrons, } \\
Z & =\frac{\nu}{2 \pi f},
\end{aligned}
$$
particles.

$\nu=$ collision frequency of electrons with heavy

If a capacitor of arbitrary configuration with a free space capacitance $C_{0}$ is inserted into this dielectric medium, a measurement of the new capacitance 
will yield a value which can be expressed as

$$
C=K C_{0} .
$$

The value of $K$ will vary with the orientation of the capacitor. To relate $\dot{K}$ to the quantities $K_{p}, K_{H}$, and $K_{T}$, we proceed as follows:

The energy stored in a capacitor is given by the expression

$$
U=\frac{C V^{2}}{2}
$$

where $V$ is the potential. If this capacitor is immersed in a dielectric medium, the stored energy becomes

$$
U=\frac{1}{2} \int \vec{D} \cdot \vec{E} d \tau=\frac{C V^{2}}{2}
$$

so that

$$
C=\frac{\int \vec{D} \cdot \vec{E} d \tau}{V^{2}}
$$

In free space

$$
\begin{aligned}
C_{0} & =\frac{\int \vec{E} \cdot \vec{E} d \tau}{V^{2}}, \\
\therefore \frac{C}{C_{0}} & =\frac{\int \vec{D} \cdot \vec{E} d \tau}{\int E^{2} d \tau} .
\end{aligned}
$$

ExpandingTthe scalar product $D \cdot E$, we have

$$
\begin{aligned}
\vec{D} \cdot \vec{E}= & \begin{array}{ccc}
K_{T} & j K_{H} & 0 \\
-j K_{H} & K_{T} & 0 \\
0 & 0 & K_{p}
\end{array} \mid\left(\begin{array}{c}
E_{x} \\
E_{y} \\
E_{z}
\end{array}\right)\left(\begin{array}{c}
E_{x} \\
E_{y} \\
E_{z}
\end{array}\right) \\
= & \left(K_{T} E_{x}+j K_{H} E_{y}\right) E_{x}+\left(-j K_{H} E_{x}\right. \\
& \left.+K_{T} E_{y}\right) E_{y}+K_{p} E_{z} E_{z} \\
= & {\left[K_{T}+\left(K_{p}-K_{T}\right) \cos ^{2} \psi\right] E^{2}, }
\end{aligned}
$$

where $\psi$ is the angle between the electric field direction and the magnetic field direction. For a uniform dielectric (i.e., one in which $K_{T}$ and $K_{p}$ are not functions of position), we have, by using eq (A3):

$$
\begin{aligned}
\frac{C}{C_{0}} & =K_{T}+\left(K_{p}-K_{T}\right) \frac{\int E^{2} \cos ^{2} \psi d \tau}{\int E^{2} d \tau} \\
& =K_{T}+\left(K_{p}-K_{T}\right) G,
\end{aligned}
$$

where $G$ depends on the geometry of the capacitor and is less than unity. Thus, the apparent dielectric constant is

$$
K=1-\frac{X}{1-Y^{2}}+G \frac{X Y^{2}}{1-Y^{2}}
$$

where $K_{T}$ and $K_{p}$ have been replaced by appropriate expressions in terms of $X$ and $Y$, assuming that $Z=0$ (i.e., the absorption is negligible). Equation (A4) may be written

$$
K=1-X\left[1+0\left(Y^{2}\right)\right]
$$

From this relation it can be seen that a magnetic field affects the dielectric constant by a term whose magnitude is of the order of $Y^{2}$. For the conditions of the experiment described in this paper, $Y^{2}=0.04$.

The authors thank the following personnel of the Goddard Space Flight Center: G. H. Spaid and S. J. Evan for technical assistance with the instrumentation; R. E. Bourdeau and L. A. Lohr for valuable assistance during the field operations; G. P. Serbu for the rocket potential data; and J. C. Seddon for assistance in vehicle tracking. The portion of this work carried out by H. A. Whale was performed while on leave from the University of Auckland, New Zealand, and in receipt of a Senior Resident Post-Doctoral Fellowship of the National Academy of Sciences.

\section{References}

Allis, W. P., Motion of electrons and ions, Handb. der Phys. XXXI, 383-444 (Springer-Verlag, Berlin, 1956).

Bourdeau, R. E., J. E. Jackson, J. A. Kane, and G. P. Serbu, Ionospheric measurements using environmental sampling techniques, NASA Tech. Note D-491 (Sept. 1960).

Jackson, J. E., and A. D. Pickar, Performance of a rocketborne 7.75 Me transmitting antenna in the ionosphere, Upper Atmosphere Rept. 28, U.S. Naval Research Laboratory Rept. 4940 (May 1957).

Jackson, J. E., Effect of oblique propagation paths upon the NRL rocket studies of the ionosphere, Upper Atmosphere Rept. 29, U.S. Naval Research Laboratory Rept. 4960 (July 1957).

Jackson, J. E., and J. A. Kane, Measurement of ionospheric electron densities using an RF probe technique, J. Geophys. Research 64, No. 8, 1074-1075 (Aug. 1959).

Jackson, J. E., and G. H. Spaid, Ground stations for NRL rocket studies of the ionosphere, Upper Atmosphere Rept. 35, U.S. Naval Research Laboratory Rept. 5342 (Aug. 31, 1959).

Jackson, J. E., and J. A. Kane, Breakdown and detuning of transmitting antennas in the ionosphere, Upper Atmosphere Rept. 36, U.S. Naval Research Laboratory Rept. 5345 (Aug. $24,1959)$.

Jackson, J. E., and J. A. Kane, Performance of an RF impedance probe in the ionosphere, J. Geophys. Research 65 , No. 7, 2209-2210 (July 1960).

Jastrow, R., and C. A. Pearse, Atmospheric drag on the satellite, J. Geophys. Research 62, No. 3, 413-423 (Sept. 1957).

Schmerling, E. R., An easily applied method for the reduction of $h^{\prime}-f$ records to $N-h$ profiles including the effects of the earth's magnetic field, J. Atmospheric and Terrest. Phys. 12, No. 1, 8-16 (1958).

Seddon, J. C., Propagation measurements in the ionosphere with the aid of the rockets, J. Geophys. Research 58, No. 3, 323-335 (Sept. 1953).

Serbu, G. P., Results from a rocket-borne Langmuir probe experiment, NASA Tech. Note D-570 (July 1961).

Whale, H. A., The excitation of electro-acoustic waves deduced from the resistive loading of a linear antenna in the ionosphere (to be published, 1962).

(Paper 66D6-224) 